\title{
Wearable sensors can reliably quantify gait alterations associated with disability in people with progressive multiple sclerosis in a clinical setting
}

\author{
Lorenza Angelini ${ }^{1} \mathbb{D} \cdot$ William Hodgkinson ${ }^{2} \cdot$ Craig Smith $^{2} \cdot$ Jessie Moorman Dodd ${ }^{2}$ Basil Sharrack ${ }^{3}$. \\ Claudia Mazzà ${ }^{1} \cdot$ David Paling $^{4}$
}

Received: 14 January 2020 / Revised: 15 May 2020 / Accepted: 16 May 2020 / Published online: 28 May 2020

(c) The Author(s) 2020

\begin{abstract}
Gait disability in people with progressive multiple sclerosis (MS) is difficult to quantify using existing clinical tools. This study aims to identify reliable and objective gait-based biomarkers to monitor progressive multiple sclerosis (MS) in clinical settings. During routine clinical visits, 57 people with secondary progressive MS and 24 healthy controls walked for 6 minutes wearing three inertial motion sensors. Fifteen gait measures were computed from the sensor data and tested for between-session reliability, for differences between controls and people with moderate and severe MS disability, and for correlation with Expanded Disability Status Scale (EDSS) scores. The majority of gait measures showed good to excellent between-session reliability when assessed in a subgroup of 23 healthy controls and 25 people with MS. These measures showed that people with MS walked with significantly longer step and stride durations, reduced step and stride regularity, and experienced difficulties in controlling and maintaining a stable walk when compared to controls. These abnormalities significantly increased in people with a higher level of disability and correlated with their EDSS scores. Reliable and objective gait-based biomarkers using wearable sensors have been identified. These biomarkers may allow clinicians to quantify clinically relevant alterations in gait in people with progressive MS within the context of regular clinical visits.
\end{abstract}

Keywords Test-retest reliability $\cdot$ Gait analysis $\cdot$ Balance $\cdot$ Temporal parameters $\cdot$ Regularity $\cdot$ Six-minute walk

\section{Introduction}

Multiple sclerosis (MS) is a common immune-mediated inflammatory and degenerative disease of the brain and spinal cord [1]. The initial clinical course is variable, but the majority of patients either present with or transition into a progressive course, characterised by the gradual

David Paling

david.paling@nhs.net

L.Angelini@sheffield.ac.uk

William Hodgkinson

drwillhodg@gmail.com

Craig Smith

craigsmxth@gmail.com

Jessie Moorman Dodd

jefamd@gmail.com

Basil Sharrack

basil.sharrack@nhs.net

Claudia Mazzà

c.mazza@sheffield.ac.uk
Department of Mechanical Engineering and Insigneo Institute for in silico Medicine, University of Sheffield, Pam Liversidge Building, Mappin Street, Sheffield S1 3JD, UK

2 Medical School, University of Sheffield, Sheffield, UK

3 Academic Department of Neuroscience, Sheffield NIHR Neuroscience BRC, Sheffield Teaching Hospital NHS Foundation Trust, Sheffield, UK

4 Sheffield Institute of Translational Neuroscience, Sheffield Teaching Hospital NHS Foundation Trust, Sheffield, UK 
accumulation of disability independent of clinical relapses, which usually significantly affect their ability to walk. It is estimated that more than 1.3 million people have progressive MS worldwide. Currently, few disease-modifying therapies are available for this phase of the illness [1,2].

Walking related disability has a significant impact on the quality of life [3] and is rated by people with MS as one of their worst symptoms [4]. Despite its importance, it is difficult to quantify this disability within clinical and research settings [5]. Typical assessments include clinical evaluation, rating scales (e.g., Expanded Disability Status Scale (EDSS) [6], 12-item MS Walking Scale [7]), and timed or distance tests (e.g., Timed 25-foot Walk, 10-m Timed Walk, 30-m Timed Walk, 100-m Timed Walk, and 2-min or 6-min Walk Test [8]). Whilst EDSS and other composite endpoints, such as the MS Functional Composite [9], have been used in clinical trials in progressive MS, these outcome measures are insensitive to the small alterations in walking disability that accumulate over the time course of a clinical trial [10]. More sensitive measures would enable clinicians to identify people with objective evidence of progressive MS more confidently, help with clinical decisions related to prognosis and the use of disease-modifying therapies, and could serve as biomarkers of disease progression in clinical trials $[1,11]$.

In recent years there has been increasing interest in bodyworn technology for quantification of disease-related gait changes [12-18]. However, their use as part of the clinical pathway of people with progressive MS is still very limited. This is partly due to the fact that previous studies had significant heterogeneity, both from the clinical and methodological perspective, and did not have a specific focus on progressive MS, which made it unclear which set of gait measures might be able to discriminate and predict different levels of walking-related disability in these people. Gait is a complex sensorimotor activity that involves not only the spatial and temporal coordination of the lower limbs but also the coordination of the trunk and arms as well as the dynamic balance [19]. Although the latter factors are known to be affected in people with MS [20], the majority of previous studies only looked at a limited subset of gait measures based on spatio-temporal features (e.g., step or stride length, step or stride duration, and gait speed, etc.), which might allow capturing only few components of disease-specific gait impairments [21]. To broaden the scope of the assessment, gait measures like intensity, jerk, regularity, and symmetry have been proposed to characterize the overall "quality and energetic efficiency" of an individual's gait [22]. These have been successfully applied to both older adults [23], those at risk of falling [24] or affected by neurological disorders such as Parkinson's disease, where they have been found to add valuable and complementary information to traditional gait analysis [21]. In MS, the use of this approach has been limited to understanding the effects of fatigue [18] and gait changes in the real world [25], but the feasibility of employing such gait measures as tools for quantifying gait abnormalities in people affected by this condition and for integrating them into routine clinical assessments is yet to be investigated. The present study is, therefore, designed to fill in this gap.

This study sought to identify biomarkers that could allow reliable and objective characterisation of gait alterations in people with progressive MS with different levels of disability compared to healthy controls, using sensors worn on the shins and lower back whist performing a walking test during routine outpatient visits. To this end, we aimed to: (1) assess between-session reliability of a comprehensive set of gait measures in healthy adults and in people with progressive MS and (2) determine which gait measures could discriminate between people with progressive MS with different levels of disability in a clinical setting.

\section{Methods}

\section{Participants}

Fifty-seven people with secondary progressive MS and 24 healthy controls took part in this study (Table 1). Participants were recruited either from the Sheffield MS Clinic at the Royal Hallamshire Hospital (Sheffield, United Kingdom) when they attended for their routine appointments or from the Sheffield Clinical trial Unit where a cohort of people with secondary progressive MS taking part in a doubleblinded intervention-based clinical trial [26] attended for their baseline assessment. None of the patients had a relapse or change of medication in the previous 3 months and none was recovering from an infection or an intercurrent illness. None of the healthy controls had any other medical or orthopaedic condition affecting their walking.

Disability was assessed using the EDSS and people with MS were split into two subgroups around the median EDSS score. People with EDSS score between 3 and 5 were classified as being moderately disabled (moderate MS, MSm) whereas people with EDSS score higher than 5 were classified as being severely disabled (severe MS, MSs).

\section{Experimental procedure}

Participants were fitted with three tri-axial inertial sensors (OPAL, APDM Inc., Portland, OR, USA) using adjustable Velcro straps (Fig. 1). Two of these were attached on the anterior shin on the right and left with the aim to accurately estimate the temporal measures (e.g., step or stride duration) [27], and one was placed on the back overlying the fifth lumbar vertebra [15] to extract the gait quality measures related to poor balance control and altered coordination [20]. 
Table 1 Demographic and clinical characteristics of the study participants

\begin{tabular}{|c|c|c|c|c|c|}
\hline & \multirow[t]{2}{*}{ People with MS } & \multirow{2}{*}{$\begin{array}{l}\text { Healthy } \\
\text { controls }\end{array}$} & \multicolumn{3}{|l|}{ Statistics } \\
\hline & & & Ctrl vs MSm & Ctrl vs MSs & MSm vs MSs \\
\hline$N$ subjects & $\begin{array}{l}\text { MS: } 57 \\
\text { MSm: } 25 \\
\text { MSs: } 32\end{array}$ & 24 & & & \\
\hline Age [years] & $\begin{array}{l}\text { MS: } 56.0(9.3) \\
\text { MSm: } 55.8(8.2) \\
\text { MSs: } 56.2(10.2)\end{array}$ & $49.8(8.4)$ & $t(47)=-2.51, p=0.02 *$ & $t(54)=-2.48, p=0.02^{*}$ & $t(55)=-0.16, p=0.87$ \\
\hline Gender [\% female] & $\begin{array}{l}\text { MS: } 67.8 \% \\
\text { MSm: } 65.4 \% \\
\text { MSs: } 69.7 \%\end{array}$ & $66.7 \%$ & $\chi^{2}(1)=0.04, p=0.85$ & $\chi^{2}(1)=0.18, p=0.68$ & $\chi^{2}(1)=0.40, p=0.53$ \\
\hline EDSS scores & $\begin{array}{l}\text { MS: } 5.5(3.0-6.5) \\
\text { MSm: } 4.0(3.0-5.0) \\
\text { MSs: } 6.0(5.5-6.5)\end{array}$ & & & & $U=0.00, z=-6.55, p<0.001$ \\
\hline $\begin{array}{l}\text { Walking assistive } \\
\text { device [\%] }\end{array}$ & $\begin{array}{l}\text { Without: } 61 \% \\
\text { Unilateral: } 25 \% \\
\text { Bilateral: } 14 \%\end{array}$ & & & & \\
\hline
\end{tabular}

Data are represented as mean (standard deviation) if normally distributed or as median (range) if not normally distributed. EDSS Expanded Disability Status Scale, $C t r l$ healthy controls

*The 6- and 7-year average age difference between Ctrl and MSm and Ctrl and MSs, respectively, is not expected to affect gait measures within this age range [44].

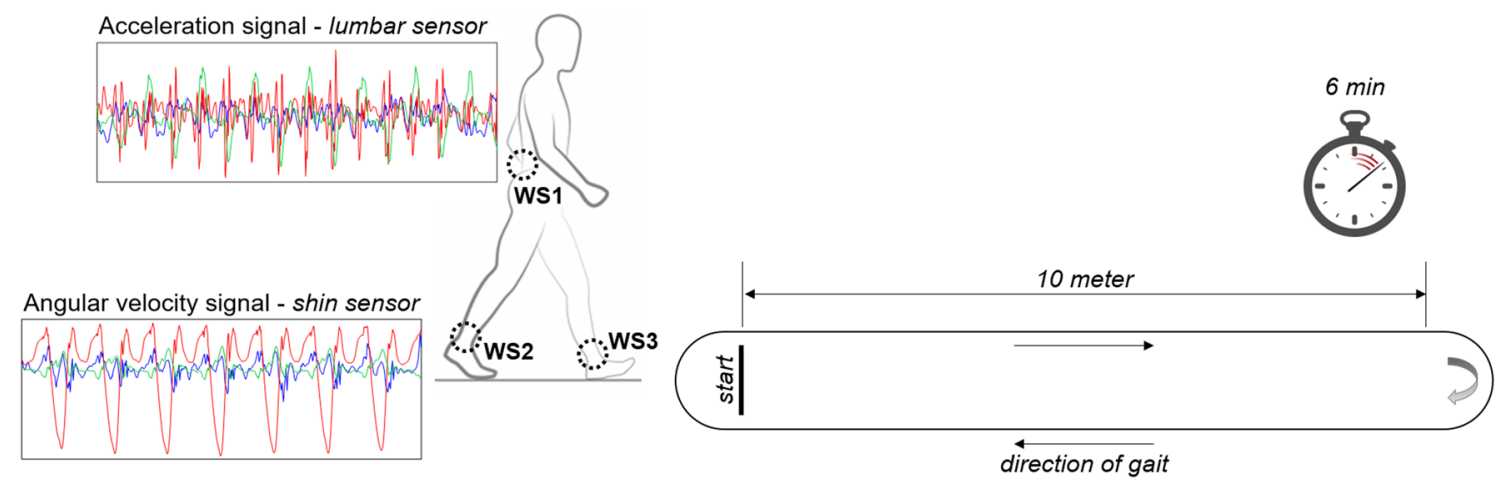

Fig. 1 Gait protocol and positioning of the wearable sensors (WS13 ). Acceleration and angular velocity signals were recorded during the walking test using three wearable sensors placed on the anterior shins and on the lower back. Typical raw acceleration and angular

Acceleration and angular velocity signals were recorded along the anatomical vertical, medio-lateral, and anteriorposterior axes at a sampling frequency of $128 \mathrm{~Hz}$ and the accelerometer range was set at $\pm 6 \mathrm{~g}$.

After being fitted with the sensors, participants were asked to walk back and forth in a hospital corridor for 6 minutes (Fig. 1). Since the test was not meant to quantify the submaximal level of functional capacity and since we aimed to propose a solution that could be widely adopted in standard hospital settings, the length of the path was limited to $10 \mathrm{~m}$, with adequate space for turning around at each end. velocity data recorded over time along anterior-posterior (AP, green line), medio-lateral (ML, red line), and vertical (V, blue line) axes are shown on the left

Participants were asked to walk at their comfortable speed and could rest and/or to use assistive devices if needed. No verbal interaction with other people was allowed during testing.

In order to assess between-session reliability, 11 people with MSm, 14 people with MSs, and 23 healthy controls repeated the assessment on a second visit, which was held 7-14 days after the first one. The sample size calculation was based on the previous work by Zou [28] and considered an expected ICC value of 0.85 with an acceptable lower limit of 0.40 , a power of $80 \%$, and a confidence interval of $95 \%$. 
Participants were assessed at the same time of the day, and patients had not experienced a relapse, change in medication, or intercurrent infection between the two assessments.

\section{Sensor data processing}

Figure 2 summarizes the main steps involved in the analysis of the sensor data (i.e., acceleration and angular velocity signals) collected during the walking test.

First, the tri-axial accelerations were reoriented to a horizontal-vertical coordinate system and filtered with a $10 \mathrm{~Hz}$ cut-off, zero phase, low-pass Butterworth filter [29]. Second, accelerations and angular velocities over 6 minutes (Fig. 2a) were segmented into walking passes (i.e., straight walking lines), with turning and resting times detected from the lumbar angular velocities [30] and discarded from subsequent analysis (Fig. 2b). Only steady-state walking passes were, therefore, processed to compute the gait measures of interest. Finally, initial floor contacts and final floor contacts of each foot were identified for each walking pass by searching for local maxima in the shin medio-lateral angular velocity of both legs (Fig. 2c) [31]. The initial contacts were referred to as Heel-Strike (i.e., the moment when the heel strikes the floor, HS), while the final contacts were referred to as ToeOff (i.e., the moment when the toe leaves the floor, TO).

\section{Gait measures}

The sensor data were processed into 15 gait measures, grouped into three domains: rhythm, variability, and balance and coordination [21, 32].
Fig. 2 Sensor data processing. a Example of angular velocity signals recorded using the lumbar and shin sensors during the walking test. b Zoom in on angular velocity signals between minute 4 and minute 5. Identification of the straight walking lines and removal of the turning times (light orange bars) from the signals. c Zoom in on one straight walking line. Detection of initial contacts (heel strike, HS, white circles) and final contacts (Toe-Off, TO, black circles) of each foot with the floor. HS occurs when heel comes in contact with the floor, while TO when toe is off the floor. HS and TO events were identified for each walking pass by finding peaks in the angular velocity of the shin sensors along the medio-lateral axis (ML, red line) (a)
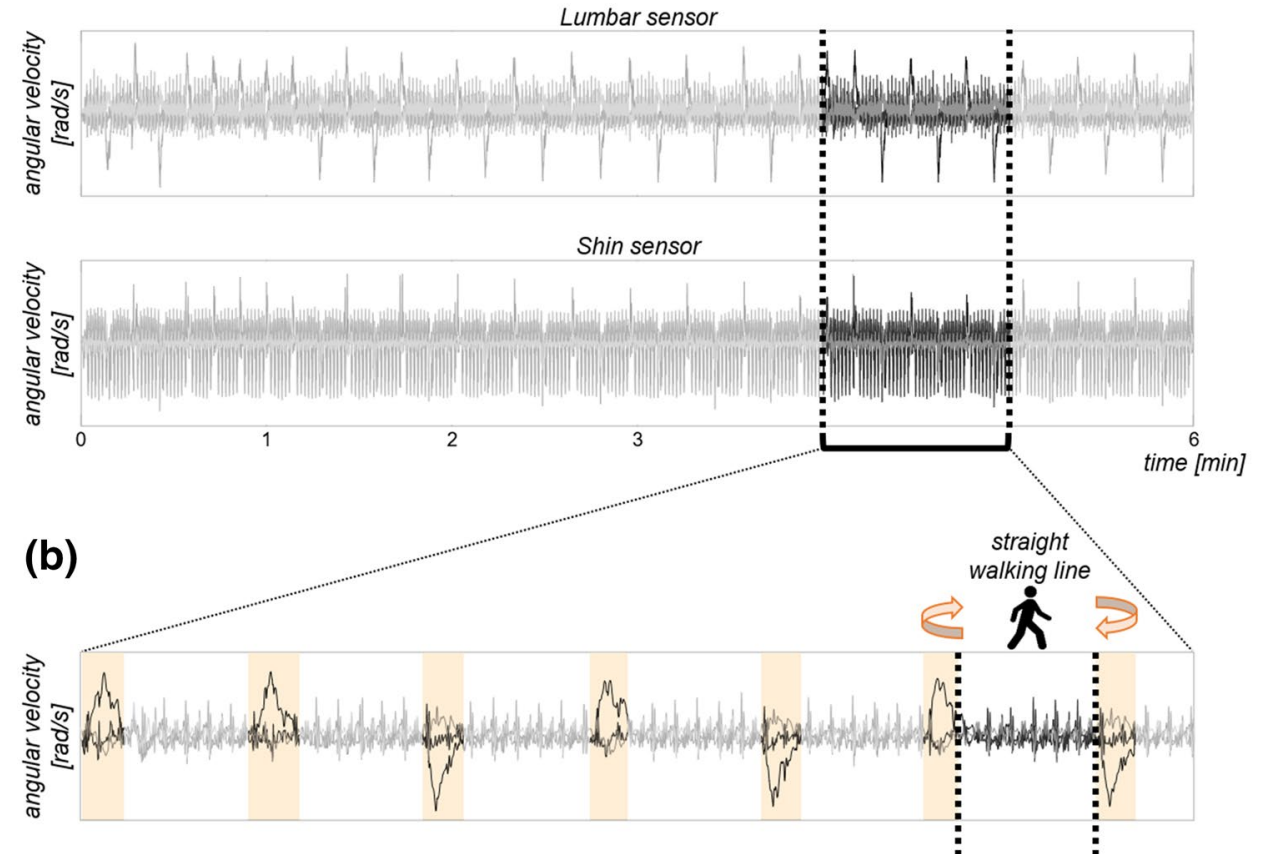

(c)

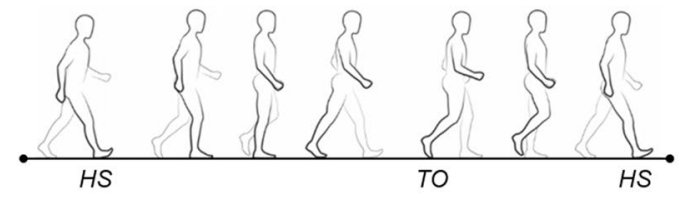


Rhythm Stride, step, stance, swing, single support, and double support durations were selected to represent the rhythm domain (Table 2). These gait measures were computed based on the timing of HS and TO events (see Fig. 2c).

Variability The variability in stride, step, stance, and swing durations were calculated, including at least 50 steps as described in Galna et al. [33]. For example, variability in stride duration was defined as the combined within-person standard deviation of the left and right stride durations (Fig. 3).

Balance and coordination Intensity, jerk, step regularity, stride regularity, and symmetry were computed from the lumbar acceleration signals for each walking pass with a minimum of five consecutive strides.

Table 3 provides a description and a visual representation of each gait measure used to characterize the gait of people with MS and healthy controls.

\section{Statistical analysis}

Participant characteristics were compared using Pearson's Chi-square for gender and independent Mann-Whitney U for age and EDSS scores. For each participant, all the gait measures were averaged over the walking time.

Between-session reliability was evaluated for participants who completed two visits. Intraclass Correlation Coefficients (ICCs) were calculated using the $\operatorname{ICC}(2, \mathrm{k})$ model with $95 \%$ confidence intervals [40]. Thresholds for ICC values were defined as per guidelines from $\mathrm{Li}$ et al. [40]: 0.75 or higher indicates excellent reliability, $0.6-0.74$ indicates good reliability, $0.4-0.59$ indicates fair reliability, and ICC lower than 0.39 indicates poor reliability. The Bland-Altman analysis was also performed to assess the agreement between the sets of gait measures obtained in the two visits [41].

Table 2 Rhythm domain measures

\section{Gait measures - Rhythm domain}

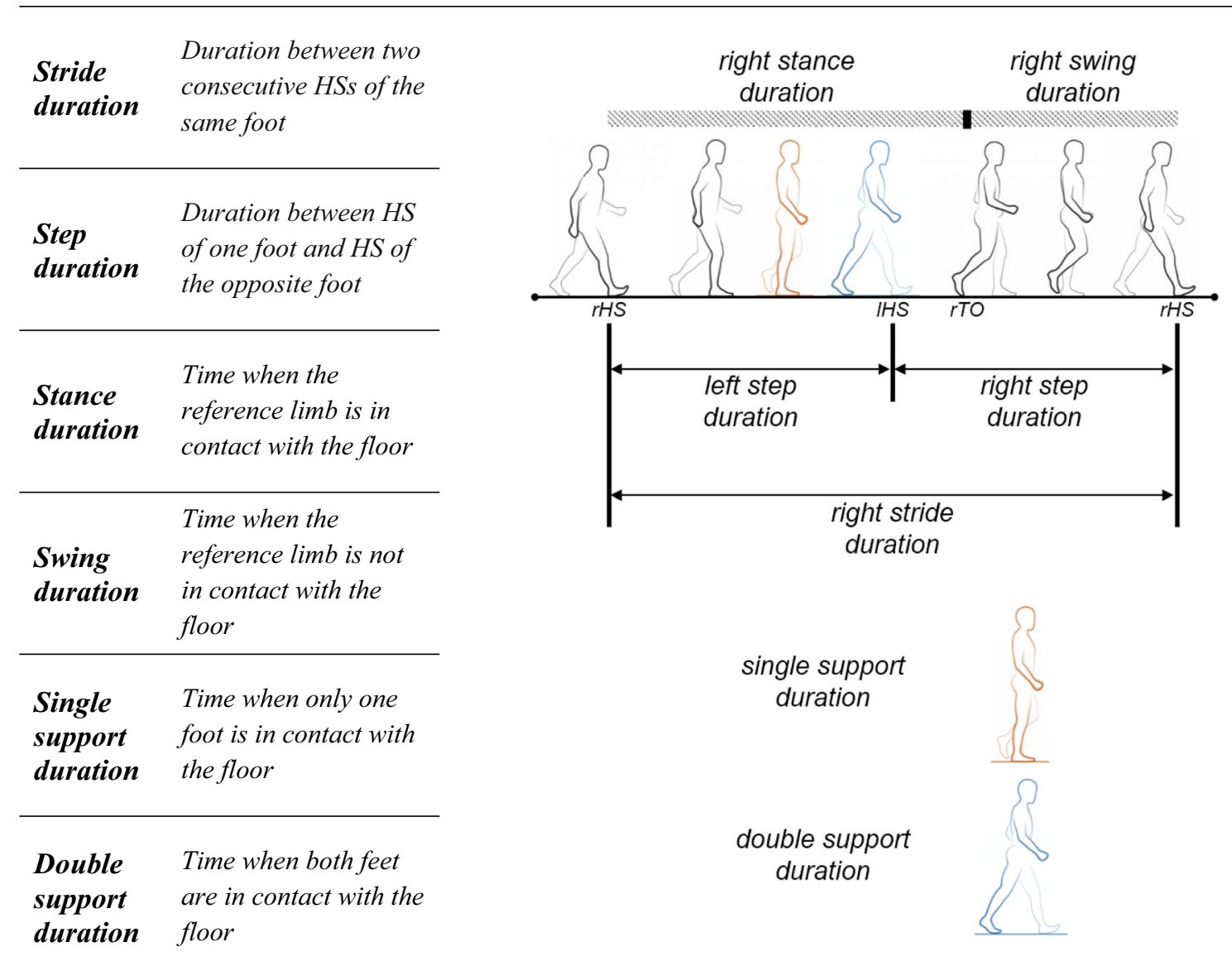

$r H S$ right heel strike, $l H S$ left heel strike, $r T O$ right toe-off 
Table 3 Balance and coordination domain measures

\section{Gait measures - Balance and coordination domain}

Intensity Intensity was calculated as the root mean square (RMS) of the acceleration modulus $\left(\right.$ acc $_{\text {mod }}=$ $\sqrt{\left.\operatorname{acc}_{A P}^{2}+\operatorname{acc}_{M L}^{2}+a c c_{V}^{2}\right)}[34-35]$.
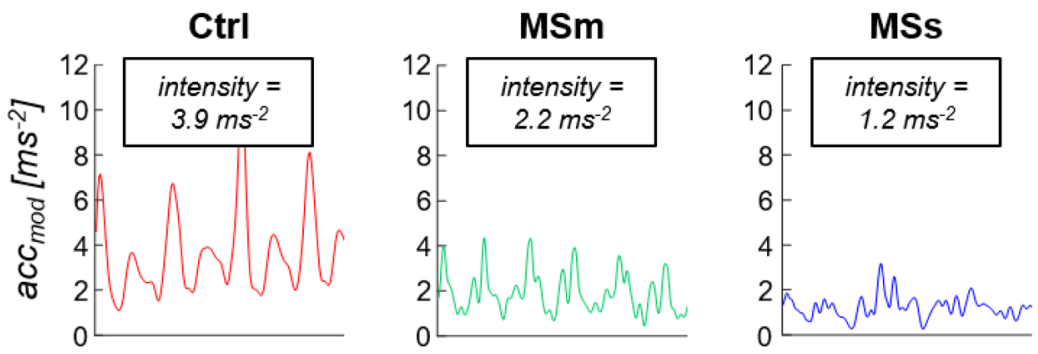

Intensity can be interpreted as a measure of upper-body dynamic balance. Decreasing values depict an increased ability for correcting postural control.

Jerk

Jerk was computed as the RMS of the jerk [36-37]. Jerk was obtained by differentiating accmod with respect to time $\left(j e r k=d_{a c c}\right.$ mod $\left./ d t\right)$.
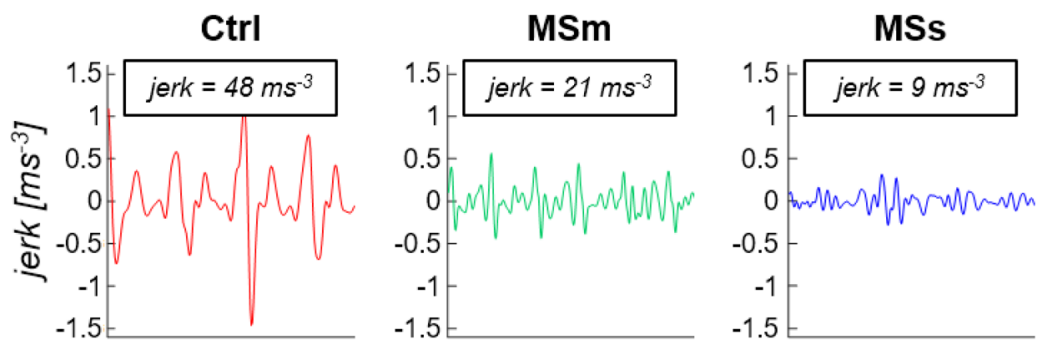

Lower values of this measure reflect a higher ability to effectively pre-plan motor strategies, resulting in a reduced likelihood to fall.

Step regularity (Ad1)

\section{Stride \\ regularity \\ (Ad2)}

Ad1 and Ad2 are the first and second peak values respectively in the normalized autocorrelation function computed from the acceleration modulus [38]. Adl (white circles) quantifies the correlation between consecutive steps, while Ad2 (black circles) the correlation between consecutive strides.
Correlation between each gait measure and the EDSS score was assessed in the MS group using a Spearman's rank correlation coefficient ( $r$ ).

The non-parametric Kruskal test was performed to compare gait measures from the control, MSs and MSm groups since a Shapiro-Wilk test showed that the gait measures were not normally distributed. Where a statistically significant difference was found ( $p$-value $<0.05$ ), an independent post hoc test (Mann-Whitney $U$ Test) with Bonferroni correction was carried out at a $1 \%$ level of significance to accommodate for 
Table 3 (continued)

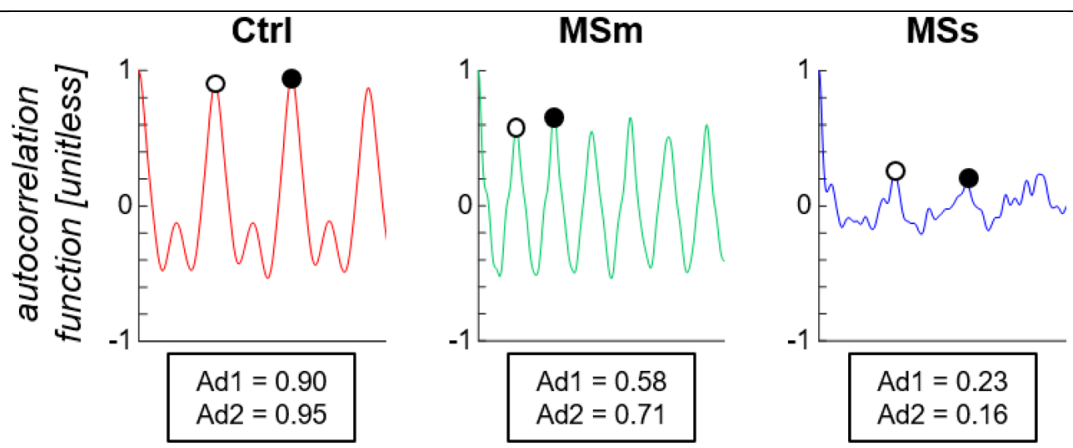

Ad1 and Ad2 measure the consistency of step-to-step and stride-to-stride pattern. Increasing Ad1 and Ad2 values, from 0 to 1 , indicate higher regularity between steps and strides, with 1 indicating perfect step and stride regularity.

Symmetry Symmetry is a measure of the correlation between left and right steps (adapted from Kobsar et al. [39]) and it is computed as follows:

$$
\text { Symmetry }=1-\frac{|\mathrm{Ad} 2-\mathrm{Ad} 1|}{\text { mean(Ad1,Ad2) }}
$$

A higher ratio implies a higher symmetry between left and right steps, with a ratio of 1 representing the perfect symmetry.

Differences in intensity, jerk, step regularity, stride regularity, and symmetry are shown for a representative healthy subject (Ctrl, red line), a representative person with moderate MS (MSm, green line), and a representative person with severe MS (MSs, blue lines).

$A P$ anterior-posterior, $M L$ medio-lateral, $V$ vertical

LOW VARIABILITY IN STRIDE DURATION

$S D$ stride duration $=20 \mathrm{~ms}$

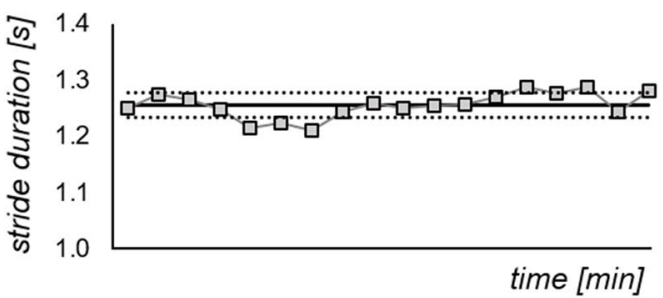

\section{HIGH VARIABILITY IN STRIDE DURATION}

$S D$ stride duration $=110 \mathrm{~ms}$

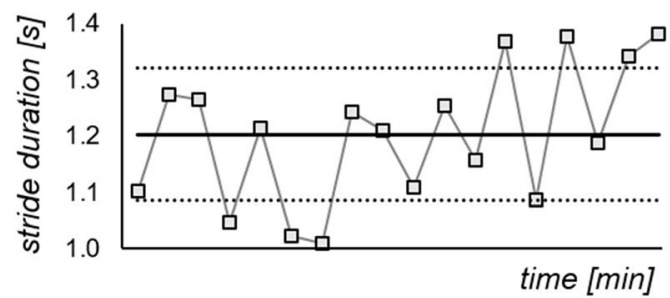

$$
S D \text { stride duration }=\sqrt{\frac{\text { variance }_{\text {left stride durations }+ \text { variance }} \text { right stride durations }}{2}}
$$

Fig. 3 Variability domain measures. Example of low and high variability (i.e., standard deviation (SD)) in stride duration

multiple comparisons (i.e., (i) people with MSm vs healthy controls, (ii) people with MSs vs healthy controls, (iii) people with MSm vs people with MSs). Median, median absolute deviation (MAD) and range values were calculated across participants for each of the investigated gait measures. 
The gait measures for people with MSm and people with MSs were normalized with respect to those for healthy controls (ctrl) by calculating the robust $z$-scores $\left(z_{\mathrm{r}},[42]\right)$ as follows:

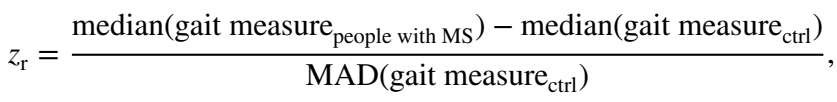

where $\operatorname{MAD}\left(\right.$ gait measure $\left.{ }_{\text {ctrl }}\right)=1.4826 \times$ median $($ Igait measure $_{\text {ctrl(i) }}-$ median(gait measure ctrl $\left._{\text {(I) }}\right) \mid$ ).

Radar plot, including the $z_{\mathrm{r}}$-score values, were used to give an overview of all the investigated gait measures and to highlight the strength of each gait measure in distinguishing people with MS with different levels of disability with respect to healthy controls. The central line in the radar plot represents healthy controls $\left(z_{\mathrm{r}}\right.$-score $\left.=0\right)$ and deviation from zero along the radial axes indicates how people with MSm and people with MSs differ from controls.

The effect size (d) for non-parametric tests was also computed as follows:

$d=\frac{z}{\sqrt{\mathrm{N}}}$

where $z$ is the $z$-score, and $\mathrm{N}$ is the number of total observations on which $z$ is based. Thresholds of $0.1,0.3$, and 0.5 were recommended by Cohen [43] for small, medium, and large effect sizes, respectively.

\section{Results}

\section{Participant characteristics}

Participant characteristics are summarized in Table 1.

\section{Between-session reliability}

Most of the gait measures demonstrated good to excellent between-session reliability (Fig. 4) for healthy controls (mean \pm standard deviation ICC: $0.88 \pm 0.08$ ), people with $\operatorname{MSm}(0.85 \pm 0.08)$, and people with MSs $(0.90 \pm 0.10)$. The Bland-Altman plots (Supplementary Fig. 1) also showed a good agreement between the sets of gait measures obtained in the two visits.

For healthy controls, 14 out of 15 gait measures showed excellent and only 1 (variability in step duration) good between-session reliability. For people with MSm, 11 out of 15 measures revealed excellent and the remaining 4 good between-session reliability. Finally, for people with MSs, 14 out of 15 measures exhibited excellent and only 1 (variability in swing duration) good between-session reliability.

\section{Gait measures}

Gait measures highlighted significant alteration in gait dynamics both in people with MSm and in people with MSs with respect to healthy controls (i.e., MSm vs healthy controls and MSs vs healthy controls), and between people with different levels of disability (i.e., MSm vs MSs) (Fig. 5).
Fig. 4 Intraclass correlation coefficients (ICCs). ICCs were calculated for healthy controls (Ctrl, red triangles), people with moderate MS (MSm, green triangles), and people with severe MS (MSs, blue triangles) who completed two testing visits, 7-14 days apart in order to evaluate the between-session reliability of each gait measure. Excellent between-session reliability is depicted in grey colour

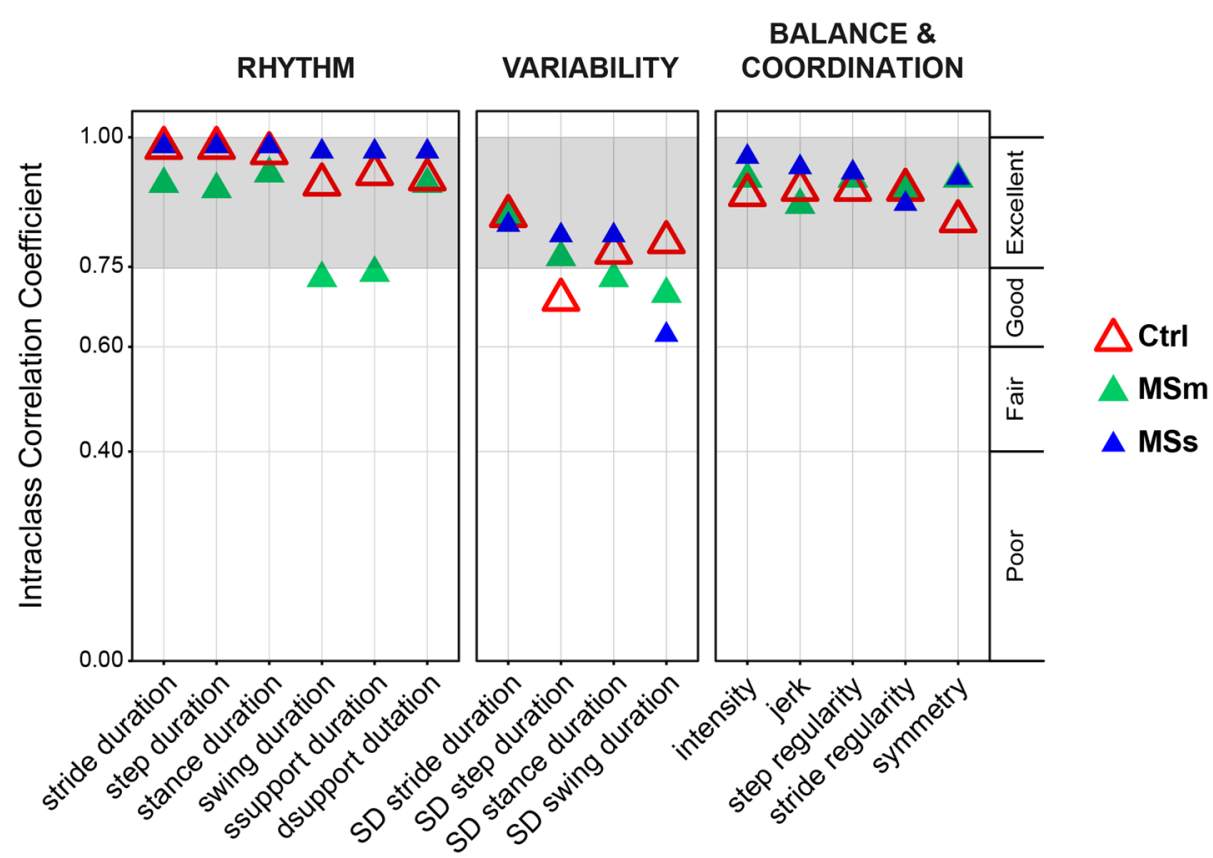




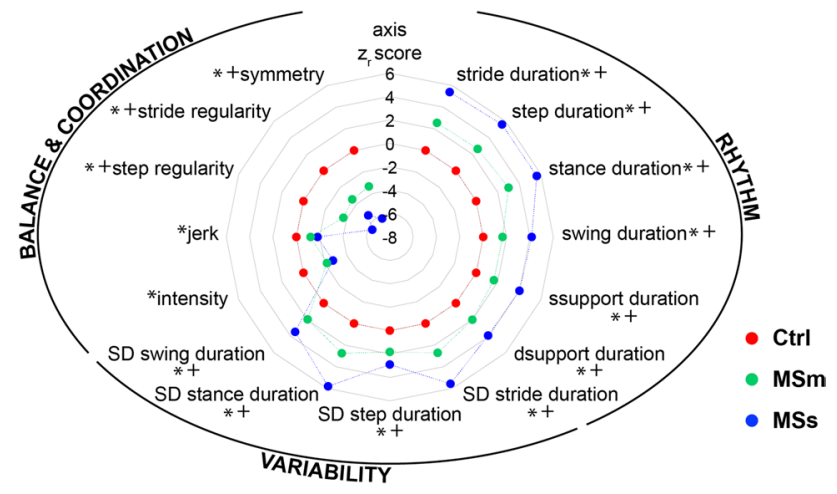

Fig. 5 Gait measures representative of rhythm, variability, and balance and coordination domains. Gait measures were calculated for healthy controls (Ctrl, red markers), people with moderate MS (MSm, green markers), and people with severe MS (MSs, blue markers). Note that increasing $z_{\mathrm{r}}$-score values in this radar plot indicate less rhythmic gait pattern (rhythm domain), more variable gait pattern (variability domain) and less difficulties in controlling balance and coordination (balance and coordination domain). $z_{\mathrm{r}}$-scores are based on median and median of absolute deviations (MAD) of Ctrl. Each radial line along the axes represents $\pm 2 \mathrm{MAD}$. Numerical values of median, MAD, and range, together with p-values and associated effect sizes, are reported in the supplementary material (Table 1). *Indicates significant differences between Ctrl and people with MSm and between Ctrl and people with MSs. + Indicates significant difference between people with MSm and people with MSs

When compared to healthy controls, gait measures showed that both people with MSm and people with MSs walked with a less rhythmic gait pattern, consisting of significantly longer step, stride, stance, swing, and single and double support durations. These measures also showed significant disruption in the normally strictly timed dynamics of HS and TO events, with increased variability seen in stride, step, stance, and swing durations (Fig. 5). Furthermore, the gait pattern in people with MSs became much less rhythmic and much more variable with respect to that observed in people with MSm (Fig. 5). This was also confirmed by the correlation between the gait measures representative of rhythm and variability domains and EDSS scores with $\mathrm{r}$ values ranging between 0.48 and 0.61 .

When additional data were leveraged from the lumbar sensor, to give a broader view of the temporal pattern of gait, our results found a reduction in intensity and jerk both in people with MSm and MSs with respect to healthy controls, indicating a higher ability to stabilize their balance with a smoother walking pattern in the presence of an impairment of the lower limbs. Our results were also able to show a decrease in the normal consistency of gait, as detected in lower values for stride regularity, step regularity, and symmetry. These differences were more marked in people with MSs than in those with MSm (Fig. 5), with values of the gait measures being on average three times larger in the former group. Weak to moderate associations ( $\mathrm{r}$ values between
0.24 and 0.46 ) were observed between the gait measures representative of balance and coordination and EDSS scores.

\section{Discussion}

Gait is complex, consisting of periodic highly sequenced and conserved movements characterised by rapid contractions and relaxations of muscle groups. By paradox, these rapid movements must propel the body forward with unvarying speed whilst simultaneously being highly energy-efficient and stable.

The pathological changes which affect the central nervous system in MS interferes with the ability of affected individuals to generate these complex movements, with eventual consequences on their walking and stability leading to falls and increasing disability.

The aim of this study was to see if small wearable sensors, integrated into the normal clinical assessment of people with MS during their routine outpatient clinic visits, were able to characterise alterations in gait that occurred in progressive MS accurately and reproducibly. Overall, the results indicated that people with MS walk at a slower pace and with a variable gait pattern of steps and strides, and have difficulties in controlling the movements of their trunk, with such impairments being more evident in people with higher degrees of disability.

Fifteen gait measures were calculated during the instrumented walking test. Almost all gait measures showed good to excellent reliability (ICC $>0.6$ ) across two separate testing days for the three groups (Fig. 4).

This study showed that ICCs in healthy controls were slightly lower than those in people with MS. Similar findings were also found in other studies [45]. A possible explanation is that since walking is not challenging for healthy adults, they often walk using a variety of normal walking patterns. In people with severe MS, some of the variables showed large confidence intervals, indicating that this subset of data might not be suitable for assessing responsiveness.

Most of the gait measures assessed in this study differed significantly between people with MS and healthy controls (Fig. 5). Alteration in the speed of gait was noted in people with MS, with longer stride and step durations, stance and swing durations and single and double support durations. These findings complement the results seen in previous studies in people with MS with moderate and severe levels of disability [14-17, 46].

Alteration in the pattern of gait was also noted, with much greater variability, in the temporal measures in people with MS (Fig. 5). Socie et al. [47] reported similar findings for variability in step duration. We also found significantly more gait variability in people with greater disability. This is consistent with the positive correlation 
between gait variability and EDSS scores reported by Socie et al. [47]. This higher gait variability could be related to fatigue, decreased muscle strength and impaired balance [47]. Moreover, gait variability could also be a marker of a fear of falling [48].

We also identified alteration in the stability of the upper body as shown by the balance and coordination domain (Fig. 5). Few studies have previously examined measures additionally derived from a sensor on the lumbar spine in people with MS [12, 13, 15, 18]. We found gait intensity to be significantly lower in people with MS compared to controls (Fig. 5). This suggests that people with MS tend to minimize upper body movement to achieve higher stability when walking with reduced leg strength $[49,50]$. Similar results are seen in normal ageing [51] and in patients following stroke [52]. In agreement with the findings of studies involving other neurological conditions [21,36], jerk was also significantly reduced in people with MS (Fig. 5) as a result of compensation strategies to maintain dynamic stability under adverse conditions. Stride and step regularity were found to be the most sensitive measures to discriminate people with MS with different levels of disability from healthy controls. People with MSm showed a significant lower step and stride regularity when compared with healthy controls, and this was increased in people with higher level of disability (i.e., people with MSs) (Fig. 5). This suggests that people with MS are less able to regulate repeating steps and strides during gait and to control the rhythmic displacements of the upper body during walking. Similar results were reported by others in MS [18] and in Parkinson's disease [21].

A possible limitation of this study, related to our decision to perform the walking test along a 10-m pathway, is the low number of strides (i.e., five strides for each straight walking pass) included for calculating step regularity, stride regularity, and symmetry. The validity of this choice is, however, supported by the existing literature. Tura et al. [53] found that the minimum number of strides needed for reliable computation of step regularity, stride regularity, and symmetry through autocorrelation sequence is between two and four steps recorded at steady-state both in healthy controls and in above-knee amputees. Additionally, Moe-Nilssen, Helbostad [38] suggested that the number of strides needed for this assessment is five in healthy adults. More recently, Angelini et al. [30] showed that, in people with MS, the gait measures we investigated in this manuscript are robust to changes in the experimental procedures, including the length of the walkway. The proposed gait measures could, therefore, be reliably integrated into the assessment performed in any clinical facility where $10-\mathrm{m}$ walk measures are being currently assessed. Additionally, focusing on the data extracted from the sensor on the lower back might also facilitate the integration of the proposed approach into continuous unsupervised mobility monitoring $[25,46,54,55]$.
Another limitation relates to the results for gait intensity and jerk. Gait velocity was not controlled for, and both people with MS and healthy controls walked at their own comfortable speed. Therefore, our findings for intensity and jerk should be interpreted with caution since they could reflect preferred gait speed [34] and/or movement amplitude and duration [56]. Whilst our protocol was delivered in a clinical environment, it did require six minutes of supervised patient walking which may be hard to replicate in an outpatient clinic setting, and would not be suitable for people at significant risk of falls. Further work will assess whether shorter walking times are able to provide the same amount of reliable information. This might also mitigate any possible limitation associated with fatigue, which we did not assess in this study.

Finally, in view of the relatively small cohort included in this study, we had to group the gait measures into three domains established according to the existing literature [21, 32]. Future studies should include factor analysis to confirm the validity of this decision. While this study was cross-sectional, we are currently longitudinally investigating a larger cohort (MOBILISE-D, www.mobilise-d.eu).

\section{Conclusions}

Our study looked at using small inertial sensors to characterise gait impairment and compensatory strategies in people with progressive MS in their normal clinical setting. This study indicates that people with progressive MS walk at a slower pace, with a much more variable pattern of steps and strides, and experience difficulties in controlling the movements of the trunk and maintaining a stable walk. These abnormalities are more prominent in people with MS with higher levels of disability. These assessments were reliable in test-retest analysis, and suitable for clinical use in monitoring patients and in research settings as accurate and responsive outcome measures for clinical trials.

Ongoing studies will expand on the cross-sectional data presented here and focus on longitudinal observation to assess the responsiveness and validate the use of the proposed gait measures as biomarkers of disease progression within the time course of clinical trials.

Acknowledgements This study was co-funded by the NIHR through the Sheffield Biomedical Research Centre (BRC, grant number ISBRC-1215-20017), by the European Union's Horizon 2020 research and innovation programme and EFPIA via the Innovative Medicine Initiative 2 (Mobilise-D project, grant number IMI22017-13-7-820820), and the UK Engineering and Physical Sciences Research Council (Multisim and MultiSim2 projects, grant numbers EP/K03877X/1 and EP/S032940/1, respectively). The views expressed are those of the author(s) and not necessarily those of the NHS, the NIHR, the Department of Health and Social Care, the IMI, the European Union, the EFPIA, or any Associated Partners. This study was carried out 
at the NIHR Sheffield Clinical Research Facility. We would like to thank all participants for giving their time to support this research and Professor Jaydip Ray.

Author contributions LA: analysis and interpretation of the data, statistical analysis, drafting the manuscript; WH, CS, JMD: data collection; BS, CM, DP: intellectual contributions to conceptualization and design of the study, interpretation of the data and critically revising the manuscript for important intellectual content.

Data availability Anonymized data can be shared upon reasonable request from other investigators. Researchers can contact Dr. David Paling (David.Paling@ @nhs.net) to gain access to the data.

\section{Compliance with ethical standards}

Conflicts of interest On behalf of all authors, the corresponding author states that there is no conflict of interest.

Ethical standards The study was approved by the NRES Committee Yorkshire \& The Humber-Bradford Leeds (reference 15/YH/0300). Written informed consents were provided by all participants prior to any testing. The project was conducted in accordance with the Declaration of Helsinki and with local ethical guidelines.

Open Access This article is licensed under a Creative Commons Attribution 4.0 International License, which permits use, sharing, adaptation, distribution and reproduction in any medium or format, as long as you give appropriate credit to the original author(s) and the source, provide a link to the Creative Commons licence, and indicate if changes were made. The images or other third party material in this article are included in the article's Creative Commons licence, unless indicated otherwise in a credit line to the material. If material is not included in the article's Creative Commons licence and your intended use is not permitted by statutory regulation or exceeds the permitted use, you will need to obtain permission directly from the copyright holder. To view a copy of this licence, visit http://creativecommons.org/licenses/by/4.0/.

\section{References}

1. Ontaneda D, Thompson AJ, Fox RJ, Cohen JA (2017) Progressive multiple sclerosis: prospects for disease therapy, repair, and restoration of function. The Lancet 389(10076):1357-1366. https ://doi.org/10.1016/S0140-6736(16)31320-4

2. Food and Drug Administration FDA and US Department of Health and Human Services US. (2017)

3. LaRocca NG (2011) Impact of Walking Impairment in Multiple Sclerosis. The Patient 4(3):189-201. https://doi. org/10.2165/11591150-000000000-00000

4. Heesen C, Böhm J, Reich C, Kasper J, Goebel M, Gold SM (2008) Patient perception of bodily functions in multiple sclerosis: gait and visual function are the most valuable. Multiple Sclerosis J 14(7):988-991. https://doi.org/10.1177/1352458508088916

5. Meyer-Moock S, Feng YS, Maeurer M, Dippel FW, Kohlmann T (2014) Systematic literature review and validity evaluation of the Expanded Disability Status Scale (EDSS) and the Multiple Sclerosis Functional Composite (MSFC) in patients with multiple sclerosis. BMC Neurol 14:58. https://doi.org/10.1186/1471-2377-14-58
6. Kurtzke JF (1983) Rating neurologic impairment in multiple sclerosis. Neurology 33(11):1444. https://doi.org/10.1212/ WNL.33.11.1444

7. Hobart JC, Riazi A, Lamping DL, Fitzpatrick R, Thompson AJ (2003) Measuring the impact of MS on walking ability. The 12-Item MS Walking Scale (MSWS-12) 60 (1):31-36. https:// doi.org/10.1212/wnl.60.1.31

8. Kieseier BC, Pozzilli C (2012) Assessing walking disability in multiple sclerosis. Multiple Sclerosis J 18(7):914-924. https:// doi.org/10.1177/1352458512444498

9. Cutter GR, Baier ML, Rudick RA, Cookfair DL, Fischer JS, Petkau J, Syndulko K, Weinshenker BG, Antel JP, Confavreux C, Ellison GW, Lublin F, Miller AE, Rao SM, Reingold S, Thompson A, Willoughby E (1999) Development of a multiple sclerosis functional composite as a clinical trial outcome measure. Brain 122(5):871-882. https://doi.org/10.1093/brain/122.5.871

10. Tur C, Montalban X (2017) Progressive MS trials: Lessons learned. Multiple Sclerosis J 23(12):1583-1592. https://doi. org/10.1177/1352458517729460

11. Inojosa H, Proschmann U, Akgün K, Ziemssen T (2019) A focus on secondary progressive multiple sclerosis (SPMS): challenges in diagnosis and definition. J Neurol. https://doi.org/10.1007/ s00415-019-09489-5

12. Huisinga JM, Mancini M, St George RJ, Horak FB (2013) Accelerometry reveals differences in gait variability between patients with multiple sclerosis and healthy controls. Ann Biomed Eng 41(8):1670-1679. https://doi.org/10.1007/s1043 9-012-0697-y

13. Craig JJ, Bruetsch AP, Lynch SG, Huisinga JM (2017) The relationship between trunk and foot acceleration variability during walking shows minor changes in persons with multiple sclerosis. Clin Biomech 49:16-21. https://doi.org/10.1016/j.clinbiomec h.2017.07.011

14. Pau M, Caggiari S, Mura A, Corona F, Leban B, Coghe G, Lorefice L, Marrosu MG, Cocco E (2016) Clinical assessment of gait in individuals with multiple sclerosis using wearable inertial sensors: Comparison with patient-based measure. Multiple Sclerosis Related Disorders 10:187-191. https://doi.org/10.1016/j.msard .2016.10.007

15. Spain RI, St. George RJ, Salarian A, Mancini M, Wagner JM, Horak FB, Bourdette D (2012) Body-worn motion sensors detect balance and gait deficits in people with multiple sclerosis who have normal walking speed. Gait Posture 35(4):573-578. https:// doi.org/10.1016/j.gaitpost.2011.11.026

16. Moon Y, McGinnis RS, Seagers K, Motl RW, Sheth N, Wright JA Jr, Ghaffari R, Sosnoff JJ (2017) Monitoring gait in multiple sclerosis with novel wearable motion sensors. PLoS ONE 12(2):e0171346. https://doi.org/10.1371/journal.pone.0171346

17. Socie MJ, Motl RW, Sosnoff JJ (2014) Examination of spatiotemporal gait parameters during the 6-min walk in individuals with multiple sclerosis. Int J Rehabil Res 37(4):311-316. https://doi. org/10.1097/MRR.0000000000000074

18. Shema-Shiratzky S, Gazit E, Sun R, Regev K, Karni A, Sosnoff JJ, Herman T, Mirelman A, Hausdorff JM (2019) Deterioration of specific aspects of gait during the instrumented 6-min walk test among people with multiple sclerosis. J Neurol 266(12):30223030. https://doi.org/10.1007/s00415-019-09500-z

19. Winter DA (2009) Biomechanics and motor control of human movement. Available via https://worldcat.org

20. Cameron MH, Nilsagard Y (2018) Chapter 15 - Balance, gait, and falls in multiple sclerosis. In: Day BL, Lord SR (eds) Handbook of Clinical Neurology, vol 159. Elsevier, pp 237-250. https://doi. org/10.1016/B978-0-444-63916-5.00015-X

21. Buckley C, Galna B, Rochester L, Mazzà C (2019) Upper body accelerations as a biomarker of gait impairment in the early stages 
of Parkinson's disease. Gait Posture 71:289-295. https://doi. org/10.1016/j.gaitpost.2018.06.166

22. Pasciuto I, Bergamini E, Iosa M, Vannozzi G, Cappozzo A (2017) Overcoming the limitations of the Harmonic Ratio for the reliable assessment of gait symmetry. J Biomechan 53:84-89. https://doi. org/10.1016/j.jbiomech.2017.01.005

23. Mazzà C, Iosa M, Pecoraro F, Cappozzo A (2008) Control of the upper body accelerations in young and elderly women during level walking. J Neuroeng Rehabil 5:30. https://doi. org/10.1186/1743-0003-5-30

24. Senden R, Savelberg HHCM, Grimm B, Heyligers IC, Meijer K (2012) Accelerometry-based gait analysis, an additional objective approach to screen subjects at risk for falling. Gait Posture 36(2):296-300. https://doi.org/10.1016/j.gaitpost.2012.03.015

25. Shema-Shiratzky S, Hillel I, Mirelman A, Regev K, Hsieh KL, Karni A, Devos H, Sosnoff JJ, Hausdorff JM (2020) A wearable sensor identifies alterations in community ambulation in multiple sclerosis: contributors to real-world gait quality and physical activity. J Neurol. https://doi.org/10.1007/s00415-020-09759-7

26. Chataway J, De Angelis F, Connick P, Parker RA, Plantone D, Doshi A, John N, Stutters J, MacManus D, Prados Carrasco F, Barkhof F, Ourselin S, Braisher M, Ross M, Cranswick G, Pavitt SH, Giovannoni G, Gandini Wheeler-Kingshott CA, Hawkins C, Sharrack B, Bastow R, Weir CJ, Stallard N, Chandran S, Gandini Wheeler-Kingshott CAM, Williams T, Beyene T, Bassan V, Zapata A, Lyle D, Cameron J, Mollison D, Colville S, Dhillon B, Gnanapavan S, Nicholas R, Rashid W, Aram J, Ford H, Overell J, Young C, Arndt H, Duddy M, Guadagno J, Evangelou N, Craner M, Palace J, Hobart J, Paling D, Kalra S, McLean B (2020) Efficacy of three neuroprotective drugs in secondary progressive multiple sclerosis (MS-SMART): a phase $2 b$, multiarm, double-blind, randomised placebo-controlled trial. Lancet Neurol 19(3):214-225. https://doi.org/10.1016/S1474 $-4422(19) 30485-5$

27. Trojaniello D, Ravaschio A, Hausdorff JM, Cereatti A (2015) Comparative assessment of different methods for the estimation of gait temporal parameters using a single inertial sensor: application to elderly, post-stroke, Parkinson's disease and Huntington's disease subjects. Gait Posture 42(3):310-316. https://doi. org/10.1016/j.gaitpost.2015.06.008

28. Zou GY (2012) Sample size formulas for estimating intraclass correlation coefficients with precision and assurance. Stat Med 31(29):3972-3981. https://doi.org/10.1002/sim.5466

29. Moe-Nilssen R (1998) A new method for evaluating motor control in gait under real-life environmental conditions. Part 1: The instrument. Clin Biomechan 13(4):320-327. https://doi.org/10.1016/ S0268-0033(98)00089-8

30. Angelini L, Carpinella I, Cattaneo D, Ferrarin M, Gervasoni E, Sharrack B, Paling D, Nair KPS, Mazza C (2019) Is a wearable sensor-based characterisation of gait robust enough to overcome differences between measurement protocols? A multi-centric pragmatic study in patients with multiple sclerosis. Sensors. https ://doi.org/10.3390/s20010079

31. Salarian A, Russmann H, Vingerhoets FJ, Dehollain C, Blanc Y, Burkhard PR, Aminian K (2004) Gait assessment in Parkinson's disease: toward an ambulatory system for long-term monitoring. IEEE Trans Biomed Eng 51:1434-1443. https://doi.org/10.1109/ tbme.2004.827933

32. Lord S, Galna B, Rochester L (2013) Moving forward on gait measurement: toward a more refined approach. Movement Disord 28(11):1534-1543. https://doi.org/10.1002/mds.25545

33. Galna B, Lord S, Rochester L (2013) Is gait variability reliable in older adults and Parkinson's disease? Towards an optimal testing protocol. Gait Posture 37(4):580-585. https://doi.org/10.1016/j. gaitpost.2012.09.025
34. Menz HB, Lord SR, Fitzpatrick RC (2003) Acceleration patterns of the head and pelvis when walking on level and irregular surfaces. Gait Posture 18(1):35-46. https://doi.org/10.1016/S0966 $-6362(02) 00159-5$

35. Moe-Nilssen R (1998) A new method for evaluating motor control in gait under real-life environmental conditions. Part 2: Gait analysis. Clin Biomechan 13(4):328-335. https://doi.org/10.1016/ S0268-0033(98)00090-4

36. Fazio P, Granieri G, Casetta I, Cesnik E, Mazzacane S, Caliandro P, Pedrielli F, Granieri E (2013) Gait measures with a triaxial accelerometer among patients with neurological impairment. Neurolog Sci 34(4):435-440. https://doi.org/10.1007/s1007 2-012-1017-x

37. Young RP, Marteniuk RG (1997) Acquisition of a multi-articular kicking task: Jerk analysis demonstrates movements do not become smoother with learning. Hum Mov Sci 16(5):677-701. https://doi.org/10.1016/S0167-9457(97)00010-9

38. Moe-Nilssen R, Helbostad JL (2004) Estimation of gait cycle characteristics by trunk accelerometry. J Biomech 37(1):121126. https://doi.org/10.1016/S0021-9290(03)00233-1

39. Kobsar D, Olson C, Paranjape R, Hadjistavropoulos T, Barden JM (2014) Evaluation of age-related differences in the strideto-stride fluctuations, regularity and symmetry of gait using a waist-mounted tri-axial accelerometer. Gait Posture 39(1):553557. https://doi.org/10.1016/j.gaitpost.2013.09.008

40. Li L, Zeng L, Lin Z-J, Cazzell M, Liu H (2015) Tutorial on use of intraclass correlation coefficients for assessing intertest reliability and its application in functional near-infrared spectroscopy-based brain imaging. J Biomed Opt 20(5):1-13. https ://doi.org/10.1117/1.Jbo.20.5.050801

41. Bland JM, Altman DG (1999) Measuring agreement in method comparison studies. Stat Methods Med Res 8(2):135-160. https ://doi.org/10.1177/096228029900800204

42. Iglewicz B, Hoaglin DC (1993) How to Detect and Handle Outliers. ASQC Quality Press

43. Cohen J (1977) CHAPTER 3 - The Significance of a Product Moment rs. In: Cohen J (ed) Statistical Power Analysis for the Behavioral Sciences. Academic Press, pp 75-107. https://doi. org/10.1016/B978-0-12-179060-8.50008-6

44. Virmani T, Gupta H, Shah J, Larson-Prior L (2018) Objective measures of gait and balance in healthy non-falling adults as a function of age. Gait Posture 65:100-105. https://doi. org/10.1016/j.gaitpost.2018.07.167

45. Craig JJ, Bruetsch AP, Lynch SG, Horak FB, Huisinga JM (2017) Instrumented balance and walking assessments in persons with multiple sclerosis show strong test-retest reliability. J NeuroEng Rehab 14(1):43. https://doi.org/10.1186/s1298 4-017-0251-0

46. Storm FA, Nair KPS, Clarke AJ, Van der Meulen JM, Mazzà C (2018) Free-living and laboratory gait characteristics in patients with multiple sclerosis. PLoS ONE 13(5):e0196463. https://doi. org/10.1371/journal.pone.0196463

47. Socie MJ, Motl RW, Pula JH, Sandroff BM, Sosnoff JJ (2013) Gait variability and disability in multiple sclerosis. Gait Posture 38(1):51-55. https://doi.org/10.1016/j.gaitpost.2012.10.012

48. Maki BE (2015) Gait Changes in Older Adults: Predictors of Falls or Indicators of Fear? J Am Geriatr Soc 45(3):313-320. https:// doi.org/10.1111/j.1532-5415.1997.tb00946.x

49. McLoughlin J, Barr C, Crotty M, Lord SR, Sturnieks DL (2015) Association of postural sway with disability status and cerebellar dysfunction in people with multiple sclerosis: a preliminary study. Int J MS Care 17(3):146-151. https://doi. org/10.7224/1537-2073.2014-003

50. DeBolt LS, McCubbin JA (2004) The effects of home-based resistance exercise on balance, power, and mobility in adults with multiple sclerosis 11 No commercial party having a direct financial 
interest in the results of the research supporting this article has or will confer a benefit on the author(s) or on any organization with which the author(s) is/are associated. Arch Phys Med Rehabil 85(2):290-297. https://doi.org/10.1016/j.apmr.2003.06.003

51. Menz HB, Lord SR, Fitzpatrick RC (2003) Age-related differences in walking stability. Age Ageing 32(2):137-142. https:// doi.org/10.1093/ageing/32.2.137

52. Mizuike C, Ohgi S, Morita S (2009) Analysis of stroke patient walking dynamics using a tri-axial accelerometer. Gait Posture 30(1):60-64. https://doi.org/10.1016/j.gaitpost.2009.02.017

53. Tura A, Rocchi L, Raggi M, Cutti AG, Chiari L (2012) Recommended number of strides for automatic assessment of gait symmetry and regularity in above-knee amputees by means of accelerometry and autocorrelation analysis. J NeuroEng Rehab 9(1):11. https://doi.org/10.1186/1743-0003-9-11

54. Motl RW, Pilutti L, Sandroff BM, Dlugonski D, Sosnoff JJ, Pula JH (2013) Accelerometry as a measure of walking behavior in multiple sclerosis. Acta Neurol Scand 127(6):384-390. https:// doi.org/10.1111/ane.12036

55. Frechette ML, Meyer BM, Tulipani LJ, Gurchiek RD, McGinnis RS, Sosnoff JJ (2019) Next Steps in Wearable Technology and Community Ambulation in Multiple Sclerosis. Curr Neurol Neurosci Rep 19(10):80. https://doi.org/10.1007/s11910-019-0997-9

56. Hogan N, Sternad D (2009) Sensitivity of smoothness measures to movement duration, amplitude, and arrests. J Mot Behav 41(6):529-534. https://doi.org/10.3200/35-09-004-rc 